\title{
Complications and mortality in a cohort of patients undergoing emergency and elective surgery with perioperative SARS-CoV-2 infection: an Italian multicenter study. Teachings of Phase 1 to be brought in Phase 2 pandemic
}

\author{
Maurizio De Luca ${ }^{1}$ - Alberto Sartori ${ }^{1}$ - Antonio Vitiello ${ }^{2}$. Giacomo Piatto ${ }^{1}$. Giulia Noaro ${ }^{1}$ - Stefano Olmi ${ }^{3}$. \\ Diego Foschi ${ }^{4}$. Luca De Re ${ }^{4}$. Marco Zappa ${ }^{5}$. Giuliano Sarro ${ }^{6}$. Umberto Rivolta ${ }^{6}$. Giorgio Giraudo ${ }^{7}$. \\ Felice Borghi ${ }^{7}$. Gabriele Pozzo ${ }^{8}$. Vincenzo Sorisio ${ }^{8}$. Giusto Pignata ${ }^{9}$. Paola Antonella Greco ${ }^{10}$. Valerio Sisti ${ }^{10}$. \\ Tommaso Campagnaro ${ }^{11}$. Alfredo Guglielmi ${ }^{11}$. Jacopo Andreuccetti ${ }^{12}$. Alberto Di Leo ${ }^{12}$. Enrico Lauro ${ }^{13}$. \\ Francesco Ricci ${ }^{13} \cdot$ Mario Musella $^{2} \cdot$ Maurizio Zizzo $^{14}$. Stefano Bonacini ${ }^{14} \cdot$ Mauro Podda $^{15} \cdot$ Adolfo Pisanu $^{15}$. \\ Pietro Coletta ${ }^{16} \cdot$ Mario Guerrieri $^{16} \cdot$ Valerio Caracino $^{17} \cdot$ Massimo Basti $^{17} \cdot$ Vincenzo Pilone $^{18} \cdot$ Marco Raffaelli $^{19}$. \\ Luigi Oragano ${ }^{20}$
}

Received: 24 August 2020 / Accepted: 21 October 2020 / Published online: 3 January 2021

(C) Italian Society of Surgery (SIC) 2021

\begin{abstract}
Since the beginning of the pandemic due to the novel severe acute respiratory syndrome coronavirus 2 (SARS-CoV-2) and its related disease, coronavirus disease 2019 (COVID-19), several articles reported negative outcomes in surgery of infected patients. Aim of this study is to report results of patients with COVID-19-positive swab, in the perioperative period after surgery. Data of COVID-19-positive patients undergoing emergent or oncological surgery, were collected in a retrospective, multicenter study, which involved 20 Italian institutions. Collected parameters were age, sex, body mass index, COVID19-related symptoms, patients' comorbidities, surgical procedure, personal protection equipment (PPE) used in operating rooms, rate of postoperative infection among healthcare staff and complications, within 30-postoperative days. 68 patients, who underwent surgery, resulted COVID-19-positive in the perioperative period. Symptomatic patients were 63 (92.5\%). Fever was the main symptom in $36(52.9 \%)$ patients, followed by dyspnoea $(26.5 \%)$ and cough $(13.2 \%)$. We recorded 22 (32\%) intensive care unit admissions, $23(33.8 \%)$ postoperative pulmonary complications and $15(22 \%)$ acute respiratory distress syndromes. As regards the ten postoperative deaths (14.7\%), 6 cases were related to surgical complications. One surgeon, one scrub nurse and two circulating nurses were infected after surgery due to the lack of specific PPE. We reported less surgery-related pulmonary complications and mortality in Sars-CoV-2-infected patients, than in literature. Emergent and oncological surgery should not be postponed, but it is mandatory to use full PPE, and to adopt preoperative screenings and strategies that mitigate the detrimental effect of pulmonary complications, mostly responsible for mortality.
\end{abstract}

Keywords SARS-CoV-2 $\cdot$ COVID-19 $\cdot$ Pulmonary complications $\cdot$ Mortality

$\begin{array}{llll}\text { Abbreviations } & \text { PPE } & \text { Personal protection equipment } \\ \text { SARS-COV-2 } & \text { Severe acute respiratory syndrome coro- } & \text { BMI } & \text { Body mass index } \\ & \text { navirus 2 } & \text { T2DM } & \text { Type II diabetes mellitus } \\ \text { COVID-19 } & \text { Coronavirus disease 2019 } & \text { ICU } & \text { Intensive care unit } \\ \text { HTN } & \text { Hypertension } & \text { ASA } & \text { American Society of Anaesthesiologists } \\ \text { CKD } & \text { Chronic kidney disease } & \text { ARDS } & \text { Acute respiratory distress syndrome }\end{array}$

Maurizio De Luca

nnwdel@tin.it

Extended author information available on the last page of the article 


\section{Introduction}

In December 2019, a novel coronavirus, initially named 2019 coronavirus (2019-nCoV) and later defined severe acute respiratory syndrome coronavirus 2 (SARS-CoV-2), emerged in Wuhan, China [1]. On 11 March 2020, the World Health Organization (WHO) declared that the coronavirus 2019 (COVID-19) outbreak could be characterized as a pandemic [2]. As of 16 August 2020, the infection had spread worldwide, with $21,294,845$ confirmed cases and 761,779 deaths [3]. Italy was the first-hit country in Europe, and due to the high transmissibility of the virus, a high rate of critical patients required intensive care unit (ICU) admission and ventilation support. In some areas, particularly in the north, epidemic spread has been greater, hospitals were overwhelmed and there were important critical issues for the treatment of Sars-CoV-2-infected patients. Surgical theatres were converted into additional ICUs, and only emergent or oncological procedures were allowed. This major health crisis has induced Italian surgical and anaesthesiologic societies to provide recommended clinical pathways for COVID-19-positive patients to improve postoperative outcomes and to reduce the risk of infection for the operating room team [4]. A high rate of complications and unexpectedly high mortality have been reported after surgery in COVID-19 subjects [5]. The aim of this paper was to collect and report the results of emergent and oncological elective surgical procedures in COVID-19-positive patients in 20 Italian institutions with special regard to postoperative pulmonary complications and mortality.

\section{Methods}

This study is a national, multicentre, retrospective study, about patients with SARS-CoV-2 infection who underwent emergent or oncological surgery during the COVID-19 pandemic, from March 5, 2020 to May 26, 2020, in 20 different institutions in Italy. This study was approved by the Ethical Medical Committee of Treviso, Italy (protocol codex Surgery COVID-19 CIAO Study, approval number 814/CE Marca). All patients, of any age and gender, who had SARS-CoV-2 infection diagnosed within 7 days before or 30 days after emergent or oncological surgery, who underwent surgery during the recruitment period, were included in the study. Those cases with negative COVID19 swab, even in the presence of respiratory symptoms, were excluded, as well as those without 30 days follow-up. Each institution was asked to provide data on the number of patients, type and outcomes of surgical techniques. We collected only routine, anonymised data with no change to clinical care pathways. After the collection of data from each institution, an Excel (Microsoft ${ }^{\mathrm{TM}}$ ) datasheet was filled with age, sex, body mass index (BMI), COVID19-related symptoms, comorbidities (hypertension, type II diabetes, pulmonary complications, other cardio-pulmonary diseases, chronic kidney disease, cancer), surgical procedure, personal protection equipment (PPE) use in the operating room, rate of postoperative infection among surgeons and nurses, 30-day complications classified according to Clavien-Dindo [6] and ASA (American Society of Anaesthesiologists) score [7]. Pulmonary complications are defined as pneumonia, pleural effusion, pulmonary embolism, acute respiratory distress syndrome (ARDS) and postoperative ventilation complications. The clinical outcomes of these surgical patients were monitored starting from March 5, 2020, to the final date of 30-day followup, June 26, 2020, when all the patients were discharged. None of the patients was diagnosed with infection after discharge. Cases with postoperative follow-up less than 30 days and/or not confirmed with nasopharyngeal swab during the hospital stay, were excluded.

\section{Statistical analysis}

The study was performed according to STROBE guidelines for observational studies [8]. Continuous variables were expressed as the mean \pm standard deviation (DS). Categorical variables were expressed as frequencies and percentages and compared by Pearson's Chi square or Fisher's exact test between ICU and non-ICU groups or survival and death groups.

\section{Results}

A total of 68 patients $(21 \mathrm{~F} / 47 \mathrm{M})$ underwent emergent and oncological surgery during the Italian lockdown in the 20 surgical institutions involved in this study. Eighteen surgeries $(26.5 \%)$ were elective oncological, and 50 surgeries (73.5\%) were performed as emergency. No comorbidities were recorded in 19 patients (27.9\%), 1 comorbidity in 30 patients $(44.2 \%)$, or more than 1 comorbidity in 19 patients (27.9\%).

The mean preoperative age was $66.8 \pm 12.3$ years (range 31-91). The mean preoperative BMI was $28 \pm 4.2 \mathrm{~kg} / \mathrm{m}^{2}$ (range 25-35). Demographics and comorbidities are summarized in Table 1. Before surgery, five patients $(7.5 \%)$ were asymptomatic for COVID-19, and 63 patients $(92.5 \%)$ were symptomatic. Fever was the main clinical symptom in 36 patients $(52.9 \%)$, cough in nine patients (13.2\%) and dyspnoea in 18 patients $(26.5 \%)$. 
Table 1 Baseline demographics and comorbidities68 Patients, 20 institutions

\begin{tabular}{ll}
\hline Age & $66.8 \pm 12.3$ (range 31-91) \\
Sex & 21 female (30\%) \\
& 47 male $(70 \%)$ \\
BMI & $28 \pm 4.2$ (range 25-35) \\
No comorbidities & $19(27.9 \%)$ \\
1 comorbidity & $30(44.2 \%)$ \\
$>1$ comorbidity & $19(27.9 \%)$ \\
Hypertension & $26(38 \%)$ \\
COPD & $6(8.8 \%)$ \\
Diabetes & $15(22 \%)$ \\
CKD & $2(2.9 \%)$ \\
Cancer & $5(7.3 \%)$ \\
Other cardio-pulmonary diseases & $14(20.5 \%)$ \\
\hline
\end{tabular}

Before surgery, the diagnosis of COVID-19 was positive in 25 patients (41.2\%). In 21 cases (30.9\%), a COVID-19 swab was performed after a median postoperative time of $11.4 \pm 9.7$ days. In 18 emergent cases $(26.5 \%)$, a preoperative COVID-19 swab was not performed before surgery.

After surgery, 26 patients (38.2\%) had pulmonary symptoms, 23 patients $(33.8 \%)$ had pulmonary complications and in 15 cases (22\%), we recorded acute respiratory distress syndrome (ARDS) (Table 2). In 22 cases (32\%), patients were admitted to the intensive care unit (ICU). Patients were discharged after a mean time of $15.1 \pm 10.2$ days. Exitus occurred in ten patients (14.7\%) after a post-operative period of $15 \pm 10.2$ days. In 2 cases, death occurred after Hartmann procedures for perforated descendent colon, 1 case after surgery for esophageal perforation, 1 case after right hemicolectomy for cecal cancer, 1 case after Miles procedure for low rectal cancer, 1 case after laparotomy for the drainage of a perirectal abscess, 1 case after nephrostomia, 1 case after ileal resection for bowel occlusion and 2 cases in patients who underwent thoracic drain positioning (Table 2). Specifically, regarding the 10 mortality cases, 6 cases were related to a surgical complication (Clavien-Dindo V), and 4 cases were not related to any surgical complication (Table 3). With reasonable certainty, we can establish, at least in 3 cases, 2 thoracic drain positioning and 1 nephrostomia, that surgeries did not affect the severe path of the disease. Indeed, the specific procedures were carried out precisely as a consequence of the effects of COVID-19 on lungs ( 2 thoracic drain positioning) and kidney (1 nephrostomia).

Fifty-four patients $(79.4 \%)$ had a postoperative complication $\leq 3$ according to Clavien-Dindo classification, and 14 patients $(20.6 \%)$ had a postoperative Clavien-Dindo $>3$, (Table 3). Postoperative outcomes regarding deaths and pulmonary complications and related to age, BMI, preoperative diagnosis, type of surgery and comorbidities are shown in Table 4.
Surgeries were carried out with full Personal Protection Equipment (PPE) worn by the entire team in the operating room, except for one asymptomatic patient undergoing an emergent procedure. No case of infection was recorded among clinicians using PPE, while one surgeon and one scrub nurse and two circulating nurses of the above-mentioned case were infected in the week following surgery.

\section{Discussion}

Since the beginning of the COVID-19 outbreak, some cases of unexpected fatality after surgery in positive patients have been published $[3,5,9]$. Lei reported $20.5 \%$ postoperative death, $44.1 \%$ ICU admission rate and $100 \%$ postoperative pulmonary complications among 34 asymptomatic COVID19 patients who underwent elective surgery [10]. In our series, we reported different results: $14.7 \%$ postoperative mortality and $32.3 \%$ ICU patients.

The COVIDSurg Collaborative group, considering an international sample of 1128 COVID-19-positive patients undergoing emergency and elective surgery, reported mortality in 268 patients (23.8\%). Pulmonary complications occurred in 577 patients $(51.25 \%)$. Thirty-day mortality in patients with pulmonary complications occurred in 219 cases (38.0\%). In the adjusted analysis, 30-day mortality was associated with a male sex, age 70 years or older [11].

A recent review by Nahshon of all published cases suggested that it was time for a global consideration of sampling of all asymptomatic patients before surgical treatment due to the potential hazardous implications of COVID-19 infection on the peri-operative course. In this report, concerning the postoperative outcomes of patients diagnosed with COVID19 during the peri-operative period, a 14/51 (27.5\%) postoperative mortality rate and severe pulmonary complications, as well as medical staff exposure and transmission, were suggested [12]. This paper confirms the rationale by whom Italy and other affected countries decided to postpone elective non-oncological surgery and allow only emergent and oncological procedures.

Our survey aimed to collect all relevant data on the perioperative period of 30 days of COVID-19-positive patients to evaluate outcomes of general surgery during the pandemic in Italy.

In our series, we recorded postoperative death in ten patients $(14.7 \%)$ after a period of $15 \pm 10.2$ days. In 22 patients $(32.3 \%)$, we recorded the ICU admission rate and postoperative pulmonary complications in 23 patients (33.8\%). Even though five patients $(7.5 \%)$ were asymptomatic before surgery and 43 patients $(63.2 \%)$ were diagnosed with Sars-CoV-2 only after surgery, our data showed results slightly better than previously published evidence. 
Table 2 Characteristics of patients

\begin{tabular}{|c|c|c|c|c|c|c|c|c|}
\hline Urgency/election & Intensive care & Post-op swap & Symptoms & ARDS & $\begin{array}{l}\text { Radiological } \\
\text { investigas- } \\
\text { tion }\end{array}$ & Clavien-Dindo & $\begin{array}{l}\text { Post-op. complica- } \\
\text { tions }\end{array}$ & Death \\
\hline Urgency & Yes & No & Fever, dispnoea & No & CT & No & No & No \\
\hline Urgency & Yes & Yes & Fever & No & CT & II & $\begin{array}{l}\text { Deiscence anastomo- } \\
\text { sis, reoperation }\end{array}$ & No \\
\hline Election & No & Yes & Fever, dispnoea & Yes & CT & $\mathrm{IVb}$ & $\begin{array}{l}\text { Sepsis, deiscence } \\
\text { anastomosis, Reop- } \\
\text { eration }\end{array}$ & Yes \\
\hline Urgency & No & No & No & No & Rx chest & No & No & No \\
\hline Urgency & No & Yes & No & No & Rx chest & No & No & No \\
\hline Urgency & Yes & Yes & Fever & Yes & CT & IVa & Sepsis & No \\
\hline Urgency & Yes & Yes & Dispnoea & No & Rx chest & No & No & No \\
\hline Urgency & No & Yes & Dispnoea & No & Rx chest & I & Wound infection & No \\
\hline Election & No & Yes & Fever, dispnoea & No & Rx chest & IIIa & Fistula & No \\
\hline Election & No & Yes & Fever & No & Rx chest & No & No & No \\
\hline Election & No & Yes & Dispnoea & No & Rx chest & No & No & No \\
\hline Urgency & No & Yes & Pneumonia & No & Rx chest & No & No & Yes \\
\hline Urgency & No & Yes & Fever, cough & No & Rx chest & No & No & No \\
\hline Election & No & Yes & $\begin{array}{l}\text { Pneumonia, fever, } \\
\text { Cough }\end{array}$ & No & $\mathrm{CT}$ & III & Fistula & No \\
\hline Urgency & Yes & Yes & Dispnoea, pneumonia & No & CT & II & Pneumonia & No \\
\hline Election & Yes & Yes & Fever, pneumonia & No & CT & II & Pneumonia & No \\
\hline Urgency & No & Yes & Fever, pneumonia & No & CT & II & Pneumonia & No \\
\hline Urgency & Yes & No & Fever, pneumonia & Yes & CT & $\mathrm{V}$ & Pneumonia & Yes \\
\hline Urgency & No & Yes & No & No & Rx chest & No & No & No \\
\hline Urgency & Yes & No & Fever, pneumonia & Yes & CT & $\mathrm{V}$ & Pneumonia & Yes \\
\hline Urgency & No & Yes & Fever, pneumonia & No & $\mathrm{CT}$ & II & Pneumonia & No \\
\hline Urgency & No & Yes & No & No & Rx chest & No & No & No \\
\hline Urgency & No & Yes & No & No & $\mathrm{CT}$ & No & No & No \\
\hline Urgency & No & Yes & Headache & No & Rx chest & No & No & No \\
\hline Urgency & Yes & No & Fever, pneumonia & Yes & CT & $\mathrm{V}$ & Pneumonia & Yes \\
\hline Urgency & No & Yes & Fever, pneumonia & No & $\mathrm{CT}$ & II & Pneumonia & No \\
\hline Election & No & Yes & Headache & No & Rx chest & No & No & No \\
\hline Election & No & Yes & Cough & No & Rx chest & No & No & No \\
\hline Urgency & Yes & No & Fever, pneumonia & No & CT & II & Pneumonia & No \\
\hline Urgency & Yes & No & Fever & No & CT & II & Wound infection & No \\
\hline Urgency & No & Yes & Fever, pneumonia & No & CT & II & Pneumonia & No \\
\hline Urgency & No & Yes & Pneumonia & No & Rx chest & $\mathrm{V}$ & bowel infarction & Yes \\
\hline Urgency & No & Yes & dispnoea & No & $\mathrm{CT}$ & No & No & No \\
\hline Election & No & Yes & dispnoea & No & Rx chest & I & Wound infection & No \\
\hline Urgency & No & Yes & Cough & No & Rx chest & No & No & No \\
\hline Urgency & No & Yes & Cough & No & Rx chest & No & No & No \\
\hline Urgency & Yes & Yes & Fever & No & Rx chest & No & No & No \\
\hline Urgency & Yes & Yes & Fever & No & Rx chest & No & No & No \\
\hline Election & No & Yes & Fever, dispnoea & No & CT & IIIa & Pulmonary embolism & No \\
\hline Election & No & Yes & Fever, cough & No & $\mathrm{CT}$ & II & Wound infection & No \\
\hline Election & No & Yes & Fever & No & CT & No & No & No \\
\hline Election & No & Yes & Fever & No & Rx chest & No & No & No \\
\hline Urgency & No & Yes & Fever & Yes & $\mathrm{CT}$ & III & Multi organ failure & Yes \\
\hline Urgency & No & Yes & Fever & No & CT & No & No & No \\
\hline Urgency & Yes & Yes & Fever & No & $\mathrm{CT}$ & II & No & No \\
\hline
\end{tabular}


Table 2 (continued)

\begin{tabular}{|c|c|c|c|c|c|c|c|c|}
\hline Urgency/election & Intensive care & Post-op swap & Symptoms & ARDS & $\begin{array}{l}\text { Radiological } \\
\text { investigas- } \\
\text { tion }\end{array}$ & Clavien-Dindo & $\begin{array}{l}\text { Post-op. complica- } \\
\text { tions }\end{array}$ & Death \\
\hline Urgency & Yes & Yes & Fever & No & $\mathrm{CT}$ & II & No & No \\
\hline Urgency & No & Yes & Fever & No & $\mathrm{CT}$ & No & No & No \\
\hline Urgency & No & Yes & Fever & No & $\mathrm{CT}$ & II & No & No \\
\hline Urgency & Yes & Yes & Fever, dispnoea & Yes & CT & III & No & No \\
\hline Urgency & No & Yes & Fever & No & CT & No & No & No \\
\hline Urgency & No & Yes & Fever & No & CT & No & No & No \\
\hline Urgency & Yes & No & Cough & No & CT & I & Wound infection & No \\
\hline Urgency & No & Yes & Pneumonia & Yes & $\mathrm{CT}$ & IV a & Sepsis, reoperation & No \\
\hline Urgency & No & No & Pneumonia & No & $\mathrm{CT}$ & IV & Respiratory failure & No \\
\hline Urgency & No & Yes & Cough & No & CT & I & Wound infection & No \\
\hline Urgency & Yes & Yes & Fever, dispnoea & Yes & $\mathrm{CT}$ & IV & Respiratory failure & No \\
\hline Urgency & No & Yes & Fever & No & Rx chest & I & Wound infection & No \\
\hline Urgency & No & No & Fever, dispnoea & Yes & Rx chest & $\mathrm{V}$ & Sepsis with shock & Yes \\
\hline Urgency & Yes & No & Fever, dispnoea & Yes & CT & IV & Sepsis, reoperation & No \\
\hline Urgency & No & Yes & Dispnoea & No & CT & II & TVP & No \\
\hline Urgency & No & Yes & Fever, dispnoea & Yes & Rx chest & $\mathrm{V}$ & Multi organ failure & Yes \\
\hline Election & Yes & Yes & Fever, dispnoea & No & Rx chest & IV & $\begin{array}{l}\text { Retroperitoneal } \\
\text { hematoma, Reop- } \\
\text { eration }\end{array}$ & No \\
\hline Election & No & Yes & $\begin{array}{l}\text { Fever, dispnoea, } \\
\text { Penumonia }\end{array}$ & Yes & $\mathrm{CT}$ & $\mathrm{V}$ & Pneumonia & Yes \\
\hline Election & Yes & Yes & Pneumonia & Yes & $\mathrm{CT}$ & II & No & No \\
\hline Urgency & No & Yes & Headache & No & Rx chest & No & No & No \\
\hline Election & Yes & Yes & Fever & Yes & CT & IV & Pneumonia & No \\
\hline Election & No & Yes & Fever & No & Rx chest & No & No & No \\
\hline Urgency & No & Yes & Cough & No & Rx chest & No & No & No \\
\hline
\end{tabular}

Table 3 Clavien-Dindo classification

\begin{tabular}{lc}
\hline Surgical complications & 19 pts. $(27.9 \%)$ \\
Clavien Dindo & \\
No complications & 34 pts. $(50.0 \%)$ \\
I & 5 pts. $(7.4 \%)$ \\
II & 10 pts. $(14.6 \%)$ \\
IIIA-IIIB & 5 pts. $(7.4 \%)$ \\
IVA-IVB & 8 pts. $(11.8 \%)$ \\
V & 6 pts. $(8.8 \%)$ \\
Death* & 10 pts. $(14.7 \%)$ \\
\hline
\end{tabular}

* Death occurred in 6 cases for surgical complications related to surgery, and 4 cases not related to surgical complications

Preoperative diagnosis did not influence the course of the surgery. However, it has affected contagiousness due to inadequate use of PPE. Our data did not record a significant difference in complication rate or mortality between emergent and elective cases. However, $70 \%$ of deaths occurred after emergent surgeries for gastrointestinal perforation in patients suffering from at least one comorbidity. Hypertension,
Table 4 Postoperative outcomes

\begin{tabular}{|c|c|c|c|c|}
\hline & 30-days mortality & $P$ & $\begin{array}{l}\text { Pulmonary } \\
\text { complica- } \\
\text { tions }\end{array}$ & $P$ \\
\hline Age & 10 patients & 0.006 & 23 patients & 0.1 \\
\hline$<70$ years & $3(4.4 \%)$ & & $16(23.5 \%)$ & \\
\hline$\geq 70$ years & $7(10.3 \%)$ & & $7(10.3 \%)$ & \\
\hline BMI & & 0.3 & & 0.2 \\
\hline$<30$ & $9(13.2 \%)$ & & $16(23.5 \%)$ & \\
\hline$\geq 30$ & $1(1.5 \%)$ & & $7(11.7 \%)$ & \\
\hline $\begin{array}{c}\text { Preoperative } \\
\text { diagnosis }\end{array}$ & & 0.8 & & 0.7 \\
\hline Yes & $7(10.3 \%)$ & & $19(27.9 \%)$ & \\
\hline No & $3(4.4 \%)$ & & $4(5.9 \%)$ & \\
\hline Type of surgery & & 0.5 & & 0.7 \\
\hline Emergency & $7(10.3 \%)$ & & $17(25.0 \%)$ & \\
\hline Elective & $3(4.4 \%)$ & & $6(8.8 \%)$ & \\
\hline Comorbidities & & 0.03 & & 0.0001 \\
\hline$<2$ & $8(11.8 \%)$ & & $16(23.5 \%)$ & \\
\hline$\geq 2$ & $2(2.9 \%)$ & & $7(10.2 \%)$ & \\
\hline
\end{tabular}


cardiovascular disease, diabetes mellitus, smoking, chronic obstructive pulmonary disease (COPD), chronic disease and malignancy were the related comorbidities for a higher postoperative rate of complications and mortality. Obesity is a known risk factor for Sars-Cov2 pulmonary complications, but in our series, body mass index (BMI) did not influence postoperative outcomes. This appears to be in contrast with a recent small case series reporting outcomes of bariatric surgery in patients with peri-operative diagnosis [13]. According to these principles, the Italian Society for Bariatric Surgery released recommendations for surgical treatment for high BMI patients during the pandemic [14].

Our data confirmed that age $>70$ years and the presence of two or more comorbidities are risk factors for negative results after surgery in COVID-19-positive patients.[15, 16] Nevertheless, elderly patients with comorbidities also have more complicated postoperative outcomes in non-infected subjects. Indeed, some authors have suggested that all previous reports regarding surgery during the COVID-19 pandemic have underestimated the effect of other variables on postoperative outcomes after surgery, such as the ASA status $[7,15,17,18]$.

Notably, even if procedures were performed in different institutions, full PPE use effectively prevented infection of the operating room team. Our survey recorded an intraoperative infection that occurred after an emergent laparoscopic case with no preoperative COVID-19 diagnosis. In this case, one surgeon, one scrub nurse and two circulating nurses were infected, and they were not wearing full PPE.

This study presents some limitations. This was a retrospective multicenter study with 68 patients, and some data remained heterogeneous.

At the beginning of the COVID-19 pandemic, the availability of nose pharyngeal swab was limited in many hospitals, and just very symptomatic patients were tested at the time of the recovery. Some patients, who needed emergent surgery, may not have been immediately tested, but subsequently underwent the nose pharyngeal swab, if COVID19-related respiratory symptoms were present.

Data collected during March 2020 could have been affected by this limited availability, and asymptomatic but COVID-19-infected patients could have been missed, suggesting that surgery-related complications and mortality rates in Sars-CoV-2 infected patients could be even lower than those reported in this series.

Then, in few weeks, the availability of tests for COVID19 diagnosis increased all over Italy, and COVID-19 swab became mandatory, both for patients already hospitalised, and for those newly recovered.

In this multicenter study, involving 20 Italian hospitals, the number of patients collected over the recruitment period, is coherent with the above-mentioned limitations. Moreover, the incidence of COVID-19 infection was very different between regions, with northern regions counting hundreds of new cases per day, and southern regions with few cases of COVID-19-postive patients, and just few needed surgery.

To date, this is the largest Italian national study of surgical operations in COVID-19-positive patients. Furthermore, data entry was very strict, rigorous and controlled.

There are many unanswered questions regarding COVID19 and surgical practice. Our data confirm the relationship between COVID-19 infection and postoperative complications and mortality even in asyntomatic patients and in elective surgery. In any case, outcomes of emergent and oncological surgery are not irreparably invalidated by COVID-19 infection. Furthermore, considering the cases of deaths in our series, we established that, in at least 3 cases, two thoracic drain positioning and onr nephrostomia, surgeries did not affect the severe path of the disease. Indeed, the specific procedures were carried out precisely as a consequence of the effects of COVID-19, respectively, on the lungs (two thoracic drain positioning) and kidney (one nephrostomia).

According to Gottlieb's study, we can distinguish in the adoption of 4-phase containment measures. The first phase was intended to slow down and stop the spread of the epidemic on a social level. The second phase is to restore social activities by transferring containment measures to the individual level. The third phase made possible by the adoption of valid therapeutic measures and/or by the acquisition of mass immunity (vaccination). The fourth phase of reworking the strategies of opposition to new pandemics [19].

An awfully long phase 2 is, therefore, expected. In Italy, we have just passed phase 1 and our institutions are in full Phase 2. For this reason, what we learned in the first phase of the full pandemic must be capitalized to make possible a safe clinical organization of phase 2 .

The evidence in medicine also shows that there are baseline rates of postoperative pulmonary complications and subsequent mortality after surgery even in a non-COVID-19 pandemic scenario.

\section{Conclusion}

The results from the Italian multicenter study show the surgery-related complications and mortality rates in SarsCoV-2-infected patients are slightly lower than in the literature. Emergent surgery should not be postponed but it is mandatory to adopt preoperative screening and the use of PPE. Pulmonary complications are mostly responsible for mortality. It is crucial to adopt strategies that mitigate the detrimental effect of pulmonary complications. Male gender, age $>70$ years and presence of 2 or more comorbidities are risk factors for negative results after surgery in COVID-19-positive patients. Even if performing surgery during the COVID-19 pandemic carries certain risks, in the 
phase 2 pandemic, the postoperative outcomes should be reinterpreted on the basis of the preoperative conditions of the patients and the type of surgery.

Funding The authors did not receive support from any organization for the submitted work.

Availability of data and material The datasets generated during and/or analysed during the current study are available from the corresponding author on reasonable request.

\section{Compliance with ethical standards}

Conflicts of interest The authors have no conflicts of interest to declare that are relevant to the content of this article.

Ethics standards Ethical approval is required for this type of study.The Ethical Medical Committee of Treviso, Italy (protocol codex Surgery COVID-19 CIAO Study, approval number 814/CE Marca) approved the study.

Human and/or animals rights This study was performed in line with the principles of the Declaration of Helsinki. This study was approved by the Ethical Medical Committee of Treviso, Italy (protocol codex Surgery COVID-19 CIAO Study, approval number 814/CE Marca).

Informed consent Anonymised data were collected, with no change to clinical care pathways. No informed consent to participate to the study was required, neither for its publication.

Consent to participate Informed consent is not required for this study.

Consent for publication Informed consent is not required for this study.

\section{References}

1. Wilder-Smith A, Chiew CJ, Lee VJ (2020) Can we contain the COVID-19 outbreak with the same measures as for SARS? Lancet Infect Dis 20(5):e102-e107. https://doi.org/10.1016/S1473 $-3099(20) 30129-8$

2. World Health Organization. Coronavirus Disease (COVID-19). Situation Report. https://www.who.int/docs/default-source/coron aviruse/situationreports/20200610-covid-19-sitrep-142.pdf?sfvrs $\mathrm{n}=180898 \mathrm{~cd} \_6$

3. World Health Organization (2020) Coronavirus Disease 2019 (COVID-19) Situation Report - 209. WHO, Geneva

4. Coccolini F, Perrone G, Chiarugi M et al (2020) Surgery in COVID-19 patients: operational directives. World J Emerg Surg 15(1):25. https://doi.org/10.1186/s13017-020-00307-2

5. Aminian A, Safari S, Razeghian-Jahromi A, Ghorbani M, Delaney CP (2020) COVID-19 outbreak and surgical practice: unexpected fatality in perioperative period. Ann Surg. https://doi.org/10.1097/ SLA.0000000000003925
6. Dindo D, Demartines N, Clavien PA (2004) Classification of surgical complications: a new proposal with evaluation in a cohort of 6336 patients and results of a survey. Ann Surg 240:205-213

7. Doyle DJ, Goyal A, Bansal P et al (2020) American society of anesthesiologistss classification (ASA Class). StatPearls Publishing LLC, StatPearls

8. Erik VE, Douglas GA, Matthias E, Stuart JP, Peter C (2007) The strengthening the reporting of observational studies in epidemiology (STROBE) statement: guidelines for reporting observational studies. Ann Int Med 147(8):573-577

9. Li YK, Peng S, Li LQ et al (2020) Clinical and transmission characteristics of Covid-19-a retrospective study of 25 cases from a single thoracic surgery department. Curr Med Sci 40:1-6. https ://doi.org/10.1007/s11596-020-2176-2

10. Lei S, Jiang F, Su W et al (2020) Clinical characteristics and outcomes of patients undergoing surgeries during the incubation period of COVID-19 infection. EClinicalMedicine. https://doi. org/10.1016/j.eclinm.2020.100331

11. Nepogodiev D, Glasbey JC, Li E (2020) Mortality and pulmonary complications in patients undergoing surgery with peri-operative SARC-COV-2 infection. An international cohort study. COVIDSurg Collab 4:1

12 Nahshon C, Bitterman A, Haddad R, Hazzan D, Lavie O (2020) Hazardous postoperative outcomes of unexpected COVID-19 infected patients: a call for global consideration of sampling all asymptomatic patients before surgical treatment. World J Surg. 2:1-5. https://doi.org/10.1007/s00268-020-05575-2

13. Aminian A, Kermansaravi M, Azizi S et al (2020) Bariatric surgical practice during the initial phase of COVID-19 outbreak. Obes Surg. https://doi.org/10.1007/s11695-020-04617-x

14 Navarra G, Komaei I, Currò G et al (2020) Bariatric surgery and the COVID-19 pandemic: SICOB recommendations on how to perform surgery during the outbreak and when to resume the activities in phase 2 of lockdown. Updates Surg. 2:1-10

15 Shakiba B, Irani S (2020) Covid-19 and perioperative mortality; where do we stand? EClinicalMedicine 22:100364

16. Kirmeier E, Eriksson LI, Lewald $\mathrm{H}$ et al (2019) Post-anaesthesia pulmonary complications after use of muscle relaxants (POPULAR): a multicentre, prospective observational study. Lancet Respir Med 7:129-140

17. Neto AS, da Costa LGV, Hemmes SNT et al (2018) The LAS VEGAS risk score for prediction of postoperative pulmonary complications: an observational study. Eur J Anaesthesiol 35:691-701

18. Tu RH, Lin JX, Li P et al (2017) Prognostic significance of postoperative pneumonia after curative resection for patients with gastric cancer. Cancer Med 6:2757-2765

19. Gottlieb S, Rivers C, McClellan MB et al (2020) National Coronavirus Response. A road map to reopening. American Enterprise Institute, Washington, D.C.

Publisher's Note Springer Nature remains neutral with regard to jurisdictional claims in published maps and institutional affiliations. 


\title{
Affiliations
}

\author{
Luigi Oragano ${ }^{20}$ \\ 1 Department of General, Oncological and Metabolic Surgery, \\ Castelfranco and Montebelluna Hospitals, Treviso, Italy \\ 2 University Federico II of Naples, Naples, Italy \\ 3 Policlinico San Marco, Bergamo, Italy \\ 4 San Giuseppe Hospital, University of the Study, Milan, Italy \\ 5 Fatebenefratelli-Sacco Hospital, Milan, Italy \\ 6 Magenta Hospital, Milan, Italy \\ 7 Santa Croce and Carle Hospital, Cuneo, Italy \\ 8 San Lazzaro Hospital, Asti, Italy \\ 9 Spedali Civili, Brescia, Italy \\ 10 San Salvatore Centrale Hospital, Pesaro, Italy \\ 11 University Borgo Roma, Verona, Italy
}

Maurizio De Luca ${ }^{1}$ (1) - Alberto Sartori ${ }^{1}$. Antonio Vitiello ${ }^{2}$. Giacomo Piatto ${ }^{1} \cdot$ Giulia Noaro $^{1}$ - Stefano Olmi ${ }^{3}$. Diego Foschi ${ }^{4}$. Luca De Re ${ }^{4}$. Marco Zappa ${ }^{5}$. Giuliano Sarro ${ }^{6}$. Umberto Rivolta ${ }^{6}$. Giorgio Giraudo ${ }^{7}$.

Felice Borghi ${ }^{7} \cdot$ Gabriele Pozzo $^{8}$. Vincenzo Sorisio ${ }^{8}$. Giusto Pignata ${ }^{9} \cdot$ Paola Antonella Greco ${ }^{10}$. Valerio Sisti ${ }^{10}$. Tommaso Campagnaro ${ }^{11}$. Alfredo Guglielmi ${ }^{11}$. Jacopo Andreuccetti ${ }^{12}$. Alberto Di Leo ${ }^{12}$. Enrico Lauro ${ }^{13}$. Francesco Ricci ${ }^{13}$. Mario Musella ${ }^{2}$. Maurizio Zizzo ${ }^{14}$. Stefano Bonacini ${ }^{14} \cdot$ Mauro Podda $^{15}$. Adolfo Pisanu ${ }^{15}$. Pietro Coletta ${ }^{16} \cdot$ Mario Guerrieri $^{16} \cdot$ Valerio Caracino $^{17} \cdot$ Massimo Basti $^{17} \cdot$ Vincenzo Pilone $^{18} \cdot$ Marco Raffaelli $^{19}$.

12 San Camillo Hospital, Trento, Italy

13 Santa Maria del Carmine Hospital, Rovereto Trento, Italy

14 Arcispedale Santa Maria Nuova, Reggio Emilia, Italy

15 University of Cagliari, Cagliari, Italy

16 Ospedali Riuniti, Ancona, Italy

17 Santo Spirito Hospital, Pescara, Italy

18 University of Salerno, Salerno, Italy

19 Policlinico Universitario Gemelli, Rome, Italy

20 San Biagio Hospital, Domodossola, Italy 